\title{
Domain patterning on non-polar cut lithium niobate by focused ion beam
}

\author{
D.S. Chezganov, A.P. Turygin, E.O. Vlasov, E.A. Pashnina, A.S. Nuraeva, V.Ya. Shur
}

School of Natural Sciences and Mathematics, Ural Federal University, 620026, Ekaterinburg, Russia chezganov.dmitry@urfu.ru

The domain evolution on the polar surfaces of lithium niobate (LN) during switching by various techniques is well studied whereas the forward growth stage of domain evolution mostly remained hidden. The domain interaction during the stage is one of the key problems for the creation of the periodical domain structures with short periods. The focused ion beam (i-beam) irradiation controlled by the lithographic system was shown to be a promising method for shortpitch domain poling due to highly localized nanosized field source induced by injected charges [1]. The study of domain switching by i-beam on the nonpolar cuts allows revealing the features of domain intergrowth along the polar direction. From a practical point of view, the geometry of the X-cut LN plates provides direct access to the largest nonlinear-optical and electro-optical tensor elements that promises a smaller size of devices.

We have studied the domain formation at the Y-cuts of 5 mol.\% MgO-doped LN induced by injected charges during focused $\mathrm{Ga}^{+}$ion irradiation in the vacuum chamber of dual-beam Auriga Crossbeam workstation (Carl Zeiss, Germany) equipped by the i-beam lithographic system (Elphy Multibeam, Germany). The irradiation of isolated dot rows with various distances was used. The static domain structures were visualized by piezoresponse force microscopy (PFM), optical microscopy and scanning electron microscopy (SEM) after selective chemical etching.

The dependences of domain sizes (length, top width, aspect ratio) on the irradiated charge, beam current and ion energy were measured. It was shown that length of needle-like domains depends linearly on dose (irradiated charge) (Fig. 1a) while domain width (width of needle top) depends as a square root. The fact was explained by the external screening of the depolarizing field by the injected charges [2]. The domain length is independent on beam current whereas the domain width demonstrated weak dependence which can be attributed to the increase of beam spot with the current.

The interaction of isolated needle-like domains was studied. It was shown that the domain length depends on the periods of the irradiated dot row. There are two distinguished situations: (1) relative large periods $(>3 \mu \mathrm{m})$, where decrease of domain length observed at edges of rows due to lower total electric field caused by the absence of adjacent dots (Fig. 1b), (2) short period $(<3 \mu \mathrm{m})$, where the interaction of charged domain wall was observed (Fig. 1c). The measurement of domain length in irradiated dot row for various spacing allowed to reveal three main regimes: (1) uniform, (2) intermitted quasiperiodic, (3) chaotic. The similar behavior was previously observed during switching by the biased tip of a scanning probe microscope [3].

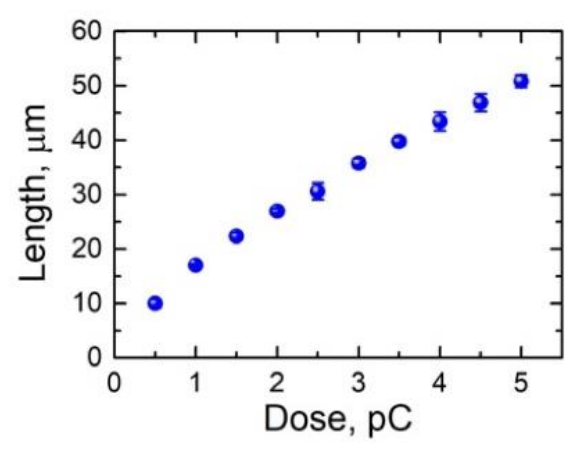

(a)

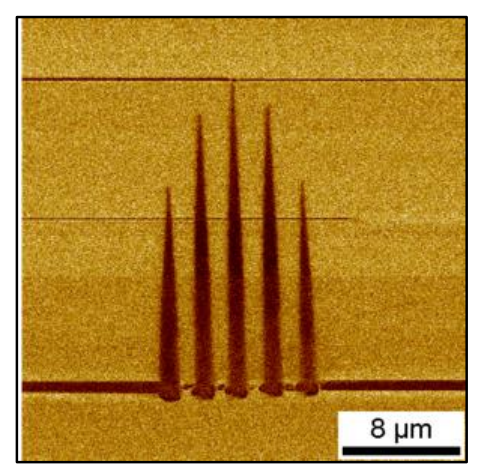

(b)

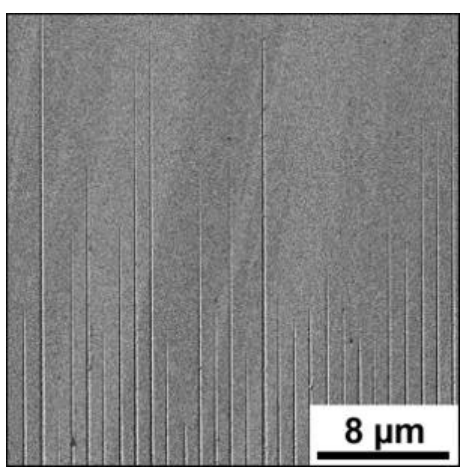

(c)

Figure 1. (a) The dependence of domain length on dose, (b) PFM image of needle-like domains, (c) SEM images of domain comb structure with $1 \mu \mathrm{m}$ spacing.

Obtained results give a new insight in the problem of the domain interaction during forward growth and can provide useful basis for application of the nanodomain engineering in development of non-linear optical frequency converters, data storage, and computing devices. 
The equipment of the Ural Center for Shared Use "Modern nanotechnology" Ural Federal University was used. The research was made possible by the Russian Science Foundation (grant № 19-72-00091).

1. D.S. Chezganov, V.Ya. Shur, E.O. Vlasov et al., Appl. Phys. Lett. 110, 082903 (2017).

2. D.S. Chezganov, E.O. Vlasov, E.A. Pashnina et al., Appl. Phys. Lett. (2019), (in press).

3. A.P. Turygin, D.O. Alikin, M.S. Kosobokov et al., ACS Appl. Mater. Interfaces 10, 36211 (2018). 